\title{
Avaliação do desempenho acadêmico de alunos do ensino fundamental em Vitória, Espírito Santo
}

\author{
Achievement performance assessment of elementary \\ school students in Vitória, Espírito Santo, Brazil
}

\author{
Tatiane Lebre DIAS \\ Sônia Regina Fiorim ENUMO² \\ Flávia Almeida TURINI ${ }^{3}$
}

\begin{abstract}
Resumo
A aquisição de habilidades acadêmicas tem sido de grande interesse para a Psicologia e para a Educação, pois problemas nessas áreas podem afetar o processo de escolarização e produzir um baixo senso de auto-eficácia para aprender. Esta pesquisa avaliou as habilidades de leitura, escrita e aritmética de alunos do ensino fundamental de Vitória, Espírito Santo, num intervalo de um ano. Participaram 172 estudantes de segunda à quinta série de uma escola pública com progressão continuada (90 alunas e 82 alunos; faixa etária: 8 a 19 anos), avaliados pelo Teste de Desempenho Escolar. Nas duas avaliações, prevaleceram a classificação inferior (1a: 56,4\%; 2a: 59,3\%) e o melhor desempenho feminino. Na segunda avaliação, aumentou significativamente a média de acertos noTeste de Desempenho Escolar (1a: 95,05; 2a: 103,2 pontos), porém não alterou a classificação. Comparando as séries nas duas avaliações, observaram-se diferenças significativas entre as séries com intervalo de dois anos. Os resultados revelam dificuldades na aquisição das habilidades acadêmicas avaliadas.
\end{abstract}

Palavras-chave: desempenho acadêmico; progressão continuada; teste de desempenho escolar.

\begin{abstract}
Academic abilities acquisition has been focused by Psychology and Education, as problems in these areas can affect schooling process and produce a low self-efficacy learning sense. This research assessed children's reading, writing and arithmetic abilities, in an Elementary School of Vitória, Espírito Santo, Brazil during oneyear. 172 (One hundred and seventy two) students from the elementary course had participated: public school children from the 2nd to 5 th grade in the context of the educational continuous progression (90 girls and 82 boys; ages: 8-19 years old) had been assessed by the School Achievement Test. In both assessments, the lowest classification prevailed (15t: 56.4\%; $\left.2^{\text {nd }}: 59.3 \%\right)$ and women got the best performance. Correct answers increased significantly in the 2nd application (1st: 95.05; 2nd: 103.2), however, the

\section{$\boldsymbol{\nabla \nu} \boldsymbol{\nabla V}$}

1 Professora Doutora, Departamento de Pedagogia, Universidade do Estado de Mato Grosso. Av. Tancredo Neves, 1095, Carvalhada, 72200-000, Cáceres, MT, Brasil. Correspondência para/Correspondenceto:T.L. DIAS.E-mail:<t.lebre@uol.com.br>

2 Professora Doutora, Departamento de Psicologia Social e do Desenvolvimento e do Programa de Pós-Graduação em Psicologia, Universidade Federal do Espírito Santo. Vitória, ES, Brasil.

3 Professora, Departamento de Psicologia e do Desenvolvimento, Universidade Federal do Espírito Santo. Vitória, ES, Brasil.

Agradecimentos: Apoio financeiro: FACITEC/Prefeitura Municipal de Vitória, Proc. n. 5012579/2001 e CNPq/MCT Proc. n. 520808/97-5 (NV) e (SU). Esta pesquisa foi realizada durante o doutorado da primeira autora, com bolsa do CNPq/MCT, orientada pela segunda autora; às bolsistas de iniciação científica Maria Júlia S. B. Pereira (PIBIC/CNPq), Juliana S. Rabbi (IC/CNPq) e Cláudia P. P. Canal (IC/CNPq) e à bolsista de aperfeiçoamento Érika S. Ferrão (FACITEC), que auxiliaram na coleta e processamento dos dados, ao Professor Romildo Rocha de Azevedo Jr., na análise estatística e na apresentação dos dados, assim como aos revisores pelas valiosas sugestões.
\end{abstract}


lowest classification hasn't change. Comparing the grades in these two applications, significant differences between the grades over an interval of two years were observed. The results disclose the difficulties in those academic abilities acquisition.

Key words: school achievement; educational continuous progression; school achievement test.

As habilidades de leitura, escrita e aritmética têm sido de grande interesse para a Psicologia porque problemas nessa área podem afetar a inclusão social e, conseqüentemente, produzir um impacto negativo sobre o autoconceito do aluno e no seu senso de auto-eficácia para aprender (Medeiros, Loureiro, Linhares \& Marturano, 2000; Stevanato, Loureiro, Linhares \& Marturano, 2003). Nesse sentido, a avaliação se mostra essencial na identificação de fatores relacionados aos problemas de aprendizagem, desde a idade precoce, tornando possível elaborar propostas de intervenção mais eficazes.

Dentre os instrumentos utilizados nos últimos anos para identificar as habilidades e competências adquiridas pelos alunos por sua participação no contexto escolar, tem-se o Sistema Nacional de Avaliação da Educação Básica (SAEB). As provas do SAEB vêm sendo aplicadas desde 1990, com intervalo de dois anos, na quarta e na oitava série do ensino fundamental e no terceiro ano do ensino médio, cujos alunos são submetidos a provas de língua portuguesa e matemática (Barreto, Pinto, Martins \& Duran, 2001).

As informações recentes do SAEB, segundo o Instituto Nacional de Estudos Pedagógicos (Instituto Nacional de Estudos e Pesquisas Educacionais, 2004), dizem respeito ao ano de 2003. Dentre os resultados divulgados, há as médias gerais de proficiência dos estudantes brasileiros nos testes de matemática e língua portuguesa. A proficiência é um dado que explicita o desempenho dos estudantes nos testes. Tais provas foram construídas a partir de uma matriz de referência, em que estão definidas as habilidades esperadas para cada um dos ciclos avaliados (primeira série a quarta série do ensino fundamental).

Os dados do SAEB de 2003 para a quarta série mostram que as médias de desempenho para língua portuguesa aumentaram em relação ao ano de 2001. Ainda assim nenhum dos estados do Brasil atingiu a média esperada para a série. O Espírito Santo teve um aumento em 2003 comparando-se ao desempenho em 2001; entretanto essa diferença não foi significativa. Os resultados de matemática indicam que não houve diferenças entre os desempenhos médios em 2001 e 2003. Assim como ocorreu em língua portuguesa, em relação à média, nenhum estado atingiu o índice esperado em matemática. O Espírito Santo teve uma queda no desempenho de matemática entre 2001 e 2003. Outro dado apontado é a diferença no desempenho entre a rede de ensino pública e a privada: somente os alunos de escolas privadas atingiram o índice de proficiência esperado (Instituto Nacional de Estudos e Pesquisas Educacionais, 2004).

Em relação aos resultados da escala de desempenho, de acordo com o sistema de classificação do SAEB, em 2001, em língua portuguesa, 59,0\% dos estudantes da quarta série se concentravam nos níveis muito crítico e crítico. Esse índice caiu para 55,0\% em 2003. Apesar dessa diminuição, apenas 4,8\% dos estudantes se encontravam na categoria adequado em 2003. Em matemática, em 2003, 51,6\% dos alunos da quarta série se concentram nas categorias muito crítico e crítico, não havendo diferenças significativas entre essas duas avaliações. No nível adequado, estavam apenas 6,4\% dos estudantes (Instituto Nacional de Estudos e Pesquisas Educacionais, 2004).

Os dados do SAEB (Instituto Nacional de Estudos e Pesquisas Educacionais, 2004) indicam também um melhor desempenho das meninas em língua portuguesa e dos meninos em matemática; no entanto as diferenças entre os gêneros em matemática foram bem menores que em português. Essa mesma tendência foi observada em avaliações internacionais, como o PISA (Bonamino, Coscarelli \& Franco, 2002). Sobre essa diferença de gênero, Carvalho (2001) ressalta a percepção diferenciada tanto dos professores quanto dos próprios alunos, que consideram as meninas "obedientes" e "organizadas", e os meninos "indisciplinados"e "descomprometidos".

De modo geral, os dados estatísticos do SAEB revelam uma série de problemas enfrentados pelo sistema educacional brasileiro. Esses problemas podem dificultar a aprendizagem dos alunos, agravando as condições daqueles com dificuldade de aprendizagem 
gerada por diversas fontes, os quais somam $25 \%$ da população escolar, segundo estimativa de Vaughn e Bos (citados por Fonseca, 1995).

Apesar de ser preocupante na educação brasileira, o desempenho acadêmico muito abaixo do esperado para a idade e para a série escolar não surpreende; ao contrário, essa é uma situação que vem de longa data e tem sido tema de estudo de pesquisadores a fim de identificar os fatores envolvidos no fracasso escolar (Patto, 1990). Os estudos que abordam o fracasso escolar no Brasil se baseiam em diferentes perspectivas: aluno, ambiente familiar, professor, condições de desenvolvimento e do ensino, investimento e políticas educacionais, entre outros (Carvalho, 2001; Carvalho, Linhares \& Martinez, 2001; Marturano, 1999; Souza, 1997).

Alguns dos fatores relacionados ao fracasso escolar têm sido destacados: prematuridade, desnutrição, problemas emocionais, desorganização do ambiente, falta de recursos para a educação, formação de professores, jornada de trabalho inadequada, além de alta rotatividade de professores, baixa remuneração e pouca motivação tanto do aluno como do professor.

Deacordo com Wechsler (1998), as características individuais do aluno relacionadas à evasão e à repetência escolar, sejam físicas, cognitivas ou de personalidade, necessitam de atenção por parte do professor, pois, entre outras coisas, influenciam um estilo de interação professor-aluno e, conseqüentemente, o clima da sala de aula.

Para agravar a situação em relação ao papel do aluno, Araújo e Schwartzman (2002) corroboram os dados de Neves e Almeida (1996) ao também mostrarem que pais e professores da rede pública consideram o aluno como o principal responsável pela repetência escolar e pelo seu péssimo desempenho. Essa atribuição do fracasso escolar ao próprio aluno parece ser reforçada no contexto de flexibilização do sistema de avaliação adotado nacionalmente nos últimos anos com a Lei de Diretrizes e Bases da Educação (LDB) no 9394, de 20/12/1996, no Capítulo Il da Educação Básica, no Artigo 29, Parágrafo V. A avaliação passou a valorizar os aspectos qualitativos do desempenho acadêmico ao longo do período letivo, possibilitando avanços nos cursos e séries e recuperação paralela nos casos de baixo rendimento escolar (geralmente denominado de sistema de ciclos, progressão continuada e progressão automática) (Brasil, 1996).
O estabelecimento desse sistema de avaliação possibilitou a implantação de alguns programas, como o Programa de Aceleração da Aprendizagem, adotado pela Secretaria Municipal de Vitória, ES, em 1998. Esse programa tem por objetivo resgatar a auto-estima, levando em consideração o desenvolvimento cognitivo e emocional e o nível de apropriação de conteúdos mínimos por alunos cuja trajetória foi marcada pelo fracasso escolar, tendo em vista a reintegração desses alunos no curso regular do ensino.

Outro exemplo desse tipo de avaliação, também adotada pelo Município de Vitória, ES, é a progressão continuada do bloco único a quarta série, em consonância com as 360 escolas do Espírito Santo que adotaram o sistema de ciclos dentro do total de 478 escolas existentes em 2002 (Menezes-Filho, Vasconcellos \& Werlang, 2005). Embora a Prefeitura Municipal não apresente documentação ou mesmo avaliação desse programa, estudos de outros Estados mostram que o índice de reprovação diminuiu significativamente. Entretanto, segundo Carvalho (2001), a progressão automática não se relaciona a uma melhor qualidade do ensino.

Assim, se nesse novo contexto que tende a favorecer o aluno, este ainda não tem bom desempenho, há uma tendência a considerar que o fracasso deve decorrer de algo pertinente ao próprio aluno.

Caberia, então, analisar os efeitos da adoção do atual sistema de avaliação escolar. Infelizmente, são escassos os estudos sobre o sistema de progressão continuada, sendo difícil avaliar o impacto sobre os alunos e professores, de acordo com Menezes-Filho et al. (2005) e Neves e Burochovitch (2004). Além disso, os estudos do SAEB são de caráter muito mais descritivo do que crítico-argumentativo, afirmam Baretto et al. (2001). Neves e Burochovitch (2004), por sua vez, avaliam que a maioria dos estudos disponíveis sobre a progressão continuada refere-se aos professores, havendo também algumas poucas pesquisas que lidam com a percepção dos alunos.

Em revisão dessa área, Neves e Burochovitch (2004) concluem que as "... investigações têm demonstrado que a implantação dos ciclos e da progressão continuada não parece ter sido suficiente para melhorar as condições de aprendizagem dos 
estudantes e, assim, resolver as verdadeiras causas da evasão e da reprovação escolar" (p.83). Ilustrando essa situação, em uma escola do interior de São Paulo, Martins (2003, p.2) encontrou 76\% das classes com problemas de alfabetização e considerou esse dado "... significativo porque, no I Ciclo (primeira a quarta), a função primordial da escola é desenvolver as habilidades que facilitam a alfabetização dos alunos". Nesse estudo, ficou evidente que "alguns alunos" (grifo da autora) permanecem na escola, saindo no final do I Ciclo com muitas deficiências de aprendizagem, apesar de participarem de todo o processo de recuperação paralela, além da recuperação feita nas férias de janeiro. Conclui a autora que é na prática que ocorre o entrave desse sistema de progressão continuada ou promoção automática.

Confirmando essa situação, Dias, Silva, Enumo e Rabbi (2005) encontraram 12,1\% de uma amostra de 264 alunos da segunda à quinta série que, surpreendentemente, erraram a escrita do próprio nome, sendo que um desses alunos cursava a quinta série e dez deles cursavam a quarta série de uma escola pública em sistema de progressão continuada.

Demo (1998) também alimenta as avaliações negativas sobre a progressão continuada, argumentando que ela escamoteia a falta de aprendizagem, podendo ainda promover a idéia de que a escola pública serve apenas para as pessoas menos favorecidas economicamente.

Assim, segundo Menezes-Filho et al. (2005, p.3), "... poder-se-ia argumentar que o desempenho dos alunos piora com a instituição de ciclos, e que esta piora pode mais que compensar o maior número de anos de estudo". Essa é uma conclusão possível diante de dados do SAEB de 2001, que esses autores analisaram. Contudo, ao avaliarem os efeitos da adoção do sistema de ciclos sobre a evasão e a aprovação na quarta série e oitava série do ensino fundamental e terceiro ano do ensino médio, esses autores concluíram que:

... a adoção da progressão continuada gerou impacto significativo sobre as escolas tratadas, tanto com relação às taxas de aprovação (aumentaram) como de evasão escolar (diminuíram) para todos os níveis de ensino estudados. Além disso, verificamos que esse impacto é mais intenso quanto maior o número de anos escolares sob o regime de ciclos, e quanto maior a duração de cada ciclo.

Em outros termos, Menezes-Filho et al. (2005) não encontraram efeito significativo da política de não-repetência sobre o desempenho escolar medido por testes de aptidão. Esses dados são coerentes com aqueles obtidos por Ferrão, Beltrão e Santos (2002) ao analisarem os dados de quarta série das escolas públicas dos Estados de São Paulo e de Minas Gerais, depois de controladas características individuais e escolares.

Na contramão desses estudos que defendem a progressão continuada, a partir de uma análise das pesquisas abordando a implantação dos ciclos no ensino fundamental, Gomes (2004) salienta que os processos de "desseriação" e de combate ao fracasso escolar sofreram significativos desgastes e distorções, visto a distância percorrida entre os centros planejadores e a execução nas escolas. Ressalta ainda a necessidade de pesquisas quantitativas que utilizem modelos variados a fim de que se possa explorar melhor a realidade educacional.

Concordando com a análise de Gomes (2004) sobre a necessidade de mais pesquisas com avaliações diferenciadas e com Menezes-Filho et al. (2005, p.2) de que "... há muita controvérsia e poucos estudos analisando os efeitos da adoção da progressão continuada", pretende-se aqui contribuir para esse debate por meio da avaliação do desempenho acadêmico de alunos do ensino fundamental de uma escola pública de uma capital da Região Sudeste funcionando no sistema de ciclos. Assim, tendo como base esse quadro geral de baixo desempenho acadêmico no ensino fundamental sob o sistema de progressão continuada, essa pesquisa avaliou as habilidades de leitura, escrita e aritmética de alunos da segunda à quinta série do ensino fundamental de uma escola pública da capital do ES, por duas vezes, com intervalo de um ano. Utilizou-se, para tanto, um instrumento brasileiro voltado à avaliação das habilidades acadêmicas básicas (leitura, escrita e aritmética), o qual tem sido aplicado também em situação de pesquisa (Dias, Enumo, Turini \& Ferrão, 2003; Linhares, Santa Maria, Escolano \& Gera, 1998; Medeiros et al. , 2000; Santa Maria \& Linhares, 1999). 


\section{Método}

\section{Participantes}

Participaram deste estudo 172 estudantes de segunda à quinta série ( $2^{\text {a }}$ série $=50 ; 3^{\text {a }}$ série $=53 ; 4^{\text {a }}$ série $=28 ; 5^{\text {a }}$ série $=41$ ), de ambos os sexos (90 alunas e 82 alunos), com idade entre 8 e 19 anos, de uma escola pública funcionando no sistema de progressão continuada, em Vitória, ES, localizada na região central da capital.

\section{Procedimentos}

Para avaliar as habilidades acadêmicas de leitura, escrita e aritmética, foi aplicado o Teste de Desempenho Escolar (TDE) (Stein, 1994)4', que possui normas brasileiras. Esse teste é composto por três subtestes: a) escrita (nome próprio e ditado de 34 palavras contextualizadas em frases); b) aritmética (solução oral de três problemas e cálculo escrito de 35 operações aritméticas); e c) leitura (reconhecimento de 70 palavras isoladas). Os subtestes de escrita e de aritmética foram aplicados em grupo, por classe escolar. O subteste de leitura foi aplicado individualmente. Os resultados foram analisados de acordo com o esperado para cada série, segundo o manual do teste, resultando nas classificações inferior médio e superior, em cada subteste e no total (soma da pontuação obtida nos subtestes). Foi feita uma primeira aplicação do teste nos alunos da segunda à quinta série no final de 2001. Após 12 meses, o instrumento foi reaplicado nos mesmos alunos, que, então, freqüentavam as séries seguintes (3a a $6^{a}$ série).

\section{Resultados}

A maioria dos alunos que, em 2001, cursavam de segunda à quinta série e, em 2002, estavam entre a terceira e a sexta série, tiveram uma classificação inferior no TDE, tanto em cada subteste (escrita, aritmética e leitura), como na média total (Tabela 1). Particularmente no subteste de leitura, houve um aumento de 16,3\% de alunos com a classificação inferior em 2002, ocorrendo o mesmo no subteste de aritmética, porém com menor porcentagem (5,8\%).

De um ano para o outro, observa-se um padrão mais constante no desempenho superior, com uma queda mais expressiva no subteste de leitura. Observa-se apenas no subteste de escrita uma melhora no desempenho médio em 2002; nos demais, houve queda de desempenho classificado como médio noTDE, sendo mais expressiva em leitura (Tabela 1).

Comparando as médias obtidas a partir dos escores brutos (pontuação obtida em cada subteste e no total do teste) nas duas avaliações (ano de 2001 e ano de 2002), vê-se que houve diferenças significativas pelo teste de "t" pareado (Tabela 2). Em outras palavras, os alunos melhoram o desempenho acadêmico nas três áreas (escrita, leitura e aritmética) de um ano para o outro, afetando o desempenho total no teste, ainda que se mantenha na classificação inferior.

Cabe lembrar que, entre a primeira e a segunda avaliação, os alunos mudaram de série escolar, de forma que o teste foi corrigido de acordo com a série em que estavam. Houve, portanto, um aumento na exigência de pontuação necessária para a classificação média.

Tabela 1. Classificação obtida por alunos de uma escola pública de ensino fundamental de Vitória, ES, nos subtestes do Teste de Desempenho Escolar (TDE) nas duas aplicações $(2001,2002)$

\begin{tabular}{|c|c|c|c|c|c|c|}
\hline \multirow{3}{*}{ Subteste } & \multicolumn{6}{|c|}{ Classificação no teste de desempenho escolar } \\
\hline & \multicolumn{2}{|c|}{ Inferior (\%) } & \multicolumn{2}{|c|}{ Médio (\%) } & \multicolumn{2}{|c|}{ Superior (\%) } \\
\hline & 2001 & 2002 & 2001 & 2002 & 2001 & 2002 \\
\hline Escrita & 67,4 & 62,8 & 23,9 & 29,6 & 8,7 & 7,6 \\
\hline Aritmética & 51,8 & 57,6 & 33,7 & 27,9 & 14,5 & 14,5 \\
\hline Leitura & 43,0 & 59,3 & 34,9 & 23,2 & 22,1 & 17,5 \\
\hline Total & 56,4 & 66,2 & 34,9 & 23,2 & 8,7 & 10,6 \\
\hline
\end{tabular}

VVV

4 OTDE foi avaliado e aprovado pelo Sistema de Avaliação de Testes Psicológicos (SATEPSI) do Conselho Federal de Psicologia em 2003 (Conselho Federal de Psicologia, 2003). 
Assim, ao mudar de série, os alunos melhoraram de modo significativo o desempenho; porém não atingiram a média esperada para sua série escolar.

No que concerne ao gênero, a partir da análise das diferenças de médias noTDE, vê-se que as meninas tiveram um desempenho significativamente superior no subteste de escrita, nas duas avaliações, e também no desempenho total em 2002 (Tabela 2).

Analisando mais detalhadamente o desempenho dos alunos segundo a série escolar em que estavam em cada avaliação, de modo a verificar mudanças no intervalo de um ano de escolarização (Tabela 3), observa-se que, nos subtestes de escrita, de aritmética e no total, houve diferenças significativas nos quatro grupos de alunos (que estavam, em 2001, na segunda, terceira, quarta e quinta série, e, em 2002, nas séries subseqüentes). No subteste de leitura, somente o grupo 4 (alunos que cursavam a quinta série em 2001 e a sexta série em 2002) apresentou diferenças significativas.

Analisando os resultados do TDE entre as séries na primeira avaliação (2001) e na segunda avaliação (2002), observam-se diferenças significativas pela análise de variância (ANOVA) nos subtestes. Pela análise a posteriori do Teste Tukey (Tabela 4), em 2001, no subteste de escrita, não ocorreram diferenças significativas entre a segunda e a terceira série e entre a terceira e a quarta série; manteve-se, portanto, um intervalo de dois anos para a identificação de diferenças no desempenho dos alunos, com exceção para a quarta e a quinta série, que apresentaram diferenças significativas entre si. No subteste de aritmética e no total do TDE, não houve diferenças significativas somente entre a terceira e a quarta série. No subteste de leitura, só houve diferenças significativas entre a segunda e a terceira série.

Na segunda avaliação do subteste de escrita (2002), não houve diferenças significativas entre a quarta e a quinta série e entre a quinta e a sexta série, mantendo-se o padrão de intervalo de dois anos para que o desempenho se diferenciasse (Tabela 4). No subteste de aritmética e no total do TDE, houve diferenças entre todas as séries, com exceção da quarta para a quinta série, repetindo, assim, o padrão encontrado em 2001. No subteste de leitura, só houve diferenças significativas entre a terceira e a sexta série e entre a quarta e a sexta série, aumentando agora o intervalo de mudança de desempenho para três anos no primeiro caso. Assim, nas duas avaliações, especialmente nos subtestes de leitura e escrita, observam-se mudanças significativas no desempenho acadêmico apenas com intervalo de dois anos entre as séries escolares.

Tabela 2. Comparação do desempenho dos alunos de uma escola pública de ensino fundamental de Vitória, ES, por subteste e por gênero nas duas aplicações $(2001,2002)$ do Teste de Desempenho Escolar (TDE).

\begin{tabular}{|c|c|c|c|c|c|c|c|}
\hline \multirow[b]{2}{*}{ Subteste } & \multirow{2}{*}{ Alunos } & \multicolumn{2}{|c|}{ Amostra geral } & \multicolumn{2}{|c|}{2001} & \multicolumn{2}{|c|}{2002} \\
\hline & & 2001 & 2002 & Masculino & Feminino & Masculino & Feminino \\
\hline \multirow[t]{4}{*}{ Escrita } & $x$ & 19,61 & 23,27 & 18,20 & 20,90 & 21,95 & 24,48 \\
\hline & s & 8,23 & 7,87 & 8,31 & 8,00 & 8,08 & 7,52 \\
\hline & $" t "$ & \multicolumn{2}{|c|}{11,82} & \multicolumn{2}{|c|}{2,12} & \multicolumn{2}{|c|}{2,12} \\
\hline & $p$ & \multicolumn{2}{|c|}{$0,00^{*}$} & \multicolumn{2}{|c|}{$0,03^{*}$} & \multicolumn{2}{|c|}{$0,03^{*}$} \\
\hline \multirow[t]{4}{*}{ Aritmética } & $x$ & 14,98 & 18,39 & 14,70 & 15,24 & 18,40 & 18,71 \\
\hline & s & 5,78 & 5,34 & 0,63 & 0,62 & 5,38 & 5,33 \\
\hline & $" t "$ & \multicolumn{2}{|c|}{12,70} & \multicolumn{2}{|c|}{0,62} & \multicolumn{2}{|c|}{0,82} \\
\hline & $p$ & \multicolumn{2}{|c|}{$0,00^{*}$} & \multicolumn{2}{|c|}{0,53} & \multicolumn{2}{|c|}{0,41} \\
\hline \multirow[t]{4}{*}{ Leitura } & $x$ & 60,53 & 61,54 & 60,04 & 60,99 & 60,44 & 62,54 \\
\hline & s & 11,36 & 8,56 & 11,42 & 11,37 & 9,42 & 7,62 \\
\hline & $" t "$ & \multicolumn{2}{|c|}{$-2,00$} & \multicolumn{2}{|c|}{0,55} & \multicolumn{2}{|c|}{1,62} \\
\hline & $p$ & \multicolumn{2}{|c|}{$0,04^{*}$} & \multicolumn{2}{|c|}{0,58} & \multicolumn{2}{|c|}{0,11} \\
\hline \multirow[t]{4}{*}{ Total } & $x$ & 95,05 & 103,26 & 92,77 & 97,13 & 99,34 & 105,73 \\
\hline & s & 21,92 & 19,31 & 22,02 & 21,76 & 21,79 & 18,30 \\
\hline & $" t "$ & \multicolumn{2}{|c|}{11,86} & \multicolumn{2}{|c|}{1,31} & \multicolumn{2}{|c|}{2,09} \\
\hline & $p$ & \multicolumn{2}{|c|}{$0,00^{*}$} & \multicolumn{2}{|c|}{0,19} & \multicolumn{2}{|c|}{$0,04^{*}$} \\
\hline
\end{tabular}


Tabela 3. Comparação dos resultados obtidos na primeira (2001) e na segunda (2002) aplicação do Teste de Desempenho Escolar (TDE), para cada grupo de alunos de uma escola pública de ensino fundamental. Vitória, ES.

\begin{tabular}{|c|c|c|c|c|c|c|c|c|c|}
\hline \multirow{2}{*}{\multicolumn{2}{|c|}{ Aplicação TDE/série }} & \multicolumn{2}{|c|}{ Grupo 1} & \multicolumn{2}{|c|}{ Grupo 2} & \multicolumn{2}{|c|}{ Grupo 3} & \multicolumn{2}{|c|}{ Grupo 4} \\
\hline & & $\begin{array}{c}2001 \\
\text { 2a série }\end{array}$ & $\begin{array}{c}2002 \\
\text { 3a série }\end{array}$ & $\begin{array}{c}2001 \\
\text { 3a série }\end{array}$ & $\begin{array}{c}2002 \\
4^{a} \text { série }\end{array}$ & $\begin{array}{c}2001 \\
\text { 4a série }\end{array}$ & $\begin{array}{c}2002 \\
\text { 5a série }\end{array}$ & $\begin{array}{c}2001 \\
\text { 5a série }\end{array}$ & $\begin{array}{c}2002 \\
\text { 6a série }\end{array}$ \\
\hline \multirow[t]{4}{*}{ Escrita } & $x$ & 15,40 & 18,66 & 19,00 & 23,15 & 20,14 & 24,53 & 25,17 & 28,19 \\
\hline & s & 6,86 & 6,22 & 8,17 & 8,77 & 8,09 & 6,83 & 6,84 & 5,77 \\
\hline & $" t "$ & \multicolumn{2}{|c|}{$-6,16$} & \multicolumn{2}{|c|}{$-7,74$} & \multicolumn{2}{|c|}{$-5,44$} & \multicolumn{2}{|c|}{$-4,34$} \\
\hline & $p$ & \multicolumn{2}{|c|}{$0,00^{*}$} & \multicolumn{2}{|c|}{$0,00^{*}$} & \multicolumn{2}{|c|}{$0,00^{*}$} & \multicolumn{2}{|c|}{$0,00^{*}$} \\
\hline \multirow[t]{4}{*}{ Aritmética } & $x$ & 9,98 & 13,76 & 14,75 & 18,85 & 15,25 & 19,07 & 21,19 & 22,97 \\
\hline & s & 3,11 & 3,35 & 3,99 & 4,12 & 5,39 & 5,05 & 4,43 & 4,50 \\
\hline & " $t$ " & \multicolumn{2}{|c|}{$-7,83$} & \multicolumn{2}{|c|}{$-9,13$} & \multicolumn{2}{|c|}{$-6,04$} & \multicolumn{2}{|c|}{$-3,09$} \\
\hline & $p$ & \multicolumn{2}{|c|}{$0,00^{*}$} & \multicolumn{2}{|c|}{$0,00^{*}$} & \multicolumn{2}{|c|}{$0,00^{*}$} & \multicolumn{2}{|c|}{$0,00^{*}$} \\
\hline \multirow[t]{4}{*}{ Leitura } & $x$ & 56,86 & 58,62 & 59,79 & 60,57 & 62,00 & 61,71 & 64,97 & 66,24 \\
\hline & s & 14,71 & 9,02 & 10,94 & 9,19 & 8,52 & 8,28 & 6,59 & 4,84 \\
\hline & $" t "$ & \multicolumn{2}{|c|}{$-1,38$} & \multicolumn{2}{|c|}{$-0,91$} & \multicolumn{2}{|c|}{0,28} & \multicolumn{2}{|c|}{$-2,36$} \\
\hline & $p$ & \multicolumn{2}{|c|}{0,17} & \multicolumn{2}{|c|}{0,36} & \multicolumn{2}{|c|}{0,78} & \multicolumn{2}{|c|}{$0,02^{*}$} \\
\hline \multirow[t]{4}{*}{ Total } & $x$ & 2,24 & 91,04 & 93,55 & 102,57 & 97,28 & 105,32 & 111,09 & 117,68 \\
\hline & s & 22,22 & 16,17 & 19,65 & 19,75 & 20,05 & 17,84 & 14,18 & 12,23 \\
\hline & $" t "$ & \multicolumn{2}{|c|}{$-5,26$} & \multicolumn{2}{|c|}{$-8,46$} & & & & \\
\hline & $p$ & & & & & & & & \\
\hline
\end{tabular}

${ }^{*} p \leq 0,05$ (teste de " $t$ " pareado).

Tabela 4. Comparação das médias obtidas em cada série escolar, nos subtestes do Teste de Desempenho Escolar (TDE), por alunos de uma escola pública de ensino fundamental de Vitória, ES, na primeira (2001) e na segunda (2002) aplicação.

\begin{tabular}{llllll}
\hline Subteste & Ano & $2^{\text {a }}$ série & $3^{\text {a série }}$ & $4^{\text {a }}$ série & $5^{\text {a }}$ série \\
\hline Escrita & 2001 & $15,40^{\text {a }}$ & $19,00^{\text {ac }}$ & $20,14^{\text {bce }}$ & $25,17^{\text {bdf }}$ \\
& $2002^{*}$ & $18,66^{\text {a }}$ & $23,15^{\text {be }}$ & $24,53^{\text {ceg }}$ & $28,19^{\text {dfg }}$ \\
\multirow{2}{*}{ Aritmética } & 2001 & $9,98^{\text {a }}$ & $14,75^{\text {bc }}$ & $15,25^{\text {bce }}$ & $21,19^{\text {bdf }}$ \\
& $2002^{*}$ & $13,76^{\text {a }}$ & $18,85^{\text {be }}$ & $19,07^{\text {ceg }}$ & $22,97^{\text {dfh }}$ \\
Leitura & 2001 & $56,86^{\text {a }}$ & $59,79^{\text {ac }}$ & $62,00^{\text {acd }}$ & $64,97^{\text {bcd }}$ \\
& $2002^{*}$ & $58,62^{\text {a }}$ & $60,56^{\text {ac }}$ & $61,77^{\text {ace }}$ & $66,24^{\text {bde }}$ \\
\hline Total & 2001 & $82,24^{\text {a }}$ & $93,55^{\text {be }}$ & $97,28^{\text {ceg }}$ & $111,10^{\text {dfh }}$ \\
& $2002^{*}$ & $91,04^{\text {a }}$ & $102,57^{\text {be }}$ & $105,32^{\text {ceg }}$ & $117,68^{\text {dfh }}$ \\
\hline
\end{tabular}

*No ano de 2002, os alunos estavam em séries subseqüentes: 3a , 4a , 5a e 6a série.

Nota: Letras iguais na mesma linha representam diferenças não significativas, enquanto letras diferentes representam diferenças significativas ao nível de $5 \%$ (teste de Tukey).

Analisando uma amostra de 122 alunos em 2001

e 131 alunos em $2002^{5}$ para uma avaliação intra-série, semelhante à realizada pelo SAEB, isto é, pela comparação da terceira, quarta e quinta série na primeira avaliação (2001) com a terceira, a quarta e a quinta série na segunda avaliação (2002), encontraram-se diferenças significativas pelo teste " $t$ "de Student, apenas no subteste de aritmética entre a quarta série de 2001 e a quarta série de 2002 (Tabela 5).

\section{Discussão}

O baixo desempenho da maioria desses alunos indica déficits na aquisição de habilidades acadêmicas que deveriam ser adquiridas ao longo das séries iniciais do ensino fundamental. Os dados mostram que, além do baixo desempenho na série inicial, após um ano de escolarização, houve aumento do escore bruto, mas não significativo a ponto de eliminar a defasagem da

V V V

s Não foram computados os dados da $2^{a}$ e da $6^{a}$ série por não haver grupo de comparação. 
Tabela 5. Comparação das médias no TDE entre alunos da 3a, 4a e 5a série de uma escola pública de Vitória, ES, nos anos de 2001 e 2002.

\begin{tabular}{|c|c|c|c|c|c|c|c|}
\hline & \multirow{2}{*}{ Séries } & \multicolumn{2}{|c|}{ 3a série } & \multicolumn{2}{|c|}{ 4a série } & \multicolumn{2}{|c|}{ 5a série } \\
\hline Subtestes & & 2001 & 2002 & 2001 & 2002 & 2001 & 2002 \\
\hline \multirow[t]{4}{*}{ Escrita } & $x$ & 19,00 & 18,70 & 20,10 & 23,20 & 25,20 & 24,50 \\
\hline & s & 8,20 & 6,20 & 8,10 & 8,80 & 6,80 & 6,80 \\
\hline & $" t "$ & \multicolumn{2}{|c|}{0,24} & \multicolumn{2}{|c|}{$-1,51$} & \multicolumn{2}{|c|}{0,38} \\
\hline & $p$ & \multicolumn{2}{|c|}{0,81} & \multicolumn{2}{|c|}{0,14} & \multicolumn{2}{|c|}{0,71} \\
\hline \multirow[t]{4}{*}{ Aritmética } & $x$ & 14,80 & 13,80 & 15,30 & 18,80 & 21,20 & 19,10 \\
\hline & s & 4,00 & 3,30 & 5,40 & 4,10 & 4,40 & 5,10 \\
\hline & $" t "$ & \multicolumn{2}{|c|}{1,37} & \multicolumn{2}{|c|}{$-3,35$} & \multicolumn{2}{|c|}{1,84} \\
\hline & $p$ & \multicolumn{2}{|c|}{0,17} & \multicolumn{2}{|c|}{$0,001^{* *}$} & \multicolumn{2}{|c|}{0,07} \\
\hline \multirow[t]{4}{*}{ Leitura } & $x$ & 59,80 & 58,60 & 62,00 & 60,60 & 65,00 & 61,70 \\
\hline & s & 10,90 & 9,00 & 8,50 & 9,20 & 6,60 & 8,30 \\
\hline & $" t "$ & \multicolumn{2}{|c|}{0,59} & \multicolumn{2}{|c|}{0,68} & \multicolumn{2}{|c|}{1,82} \\
\hline & $p$ & \multicolumn{2}{|c|}{0,56} & \multicolumn{2}{|c|}{0,50} & \multicolumn{2}{|c|}{0,07} \\
\hline \multirow[t]{4}{*}{ Total } & $x$ & 93,50 & 91,00 & 97,30 & 102,60 & 111,10 & 105,30 \\
\hline & s & 19,70 & 16,20 & 20,10 & 19,70 & 14,20 & 17,80 \\
\hline & $" t "$ & \multicolumn{2}{|c|}{0,70} & \multicolumn{2}{|c|}{$-1,14$} & \multicolumn{2}{|c|}{1,49} \\
\hline & $p$ & \multicolumn{2}{|c|}{0,48} & \multicolumn{2}{|c|}{0,26} & \multicolumn{2}{|c|}{0,14} \\
\hline
\end{tabular}

${ }^{* *} p \leq 0.01$ ( teste de " $t$ " de Student).

série anterior. Foram necessários de dois a três anos de escolarização para que ocorressem diferenças no desempenho, o qual continuou sendo majoritariamente inferior pelo TDE. Esses resultados parecem corroborar as avaliações negativas feitas ao regime de ciclos e progressão continuada por alguns estudiosos do tema (Carvalho, 2001; Gomes, 2004; Martins, 2003; Neves \& Burochovitch, 2004).

Os resultados do TDE no subteste de leitura, mostrando que apenas entre a quinta e a sexta série ocorreram diferenças significativas de um ano para outro, sugerem que há defasagem no desempenho dos alunos, o que pode comprometer a aquisição de novas habilidades nas séries seguintes. Convém lembrar que a exigência de leitura no TDE é muito menor do que a feita pelo próprio SAEB, que, por sua vez, não avalia diretamente a capacidade de leitura dos alunos brasileiros ou seu grau de letramento, na medida em que não avalia a habilidade de lidar com vários textos do cotidiano, segundo Bonamino et al. (2002). Esses dados do subteste de leitura são também coerentes com uma avaliação mais ampla feita por esses mesmos autores, que verificaram, com base nos dados do Brasil como um todo, no SAEB de 1999, um aumento geral da proficiência em língua portuguesa ao longo da escolaridade dos alunos.
Contudo esse aumento no SAEB de 1999 foi modesto quando se compara a quarta e a oitava série do ensino fundamental e a oitava com a terceira série do ensino médio:"... o que significa uma aquisição ainda muito restrita de novas habilidades e competências em língua portuguesa ao longo da escolaridade básica", segundo Bonamino et al. (2002, p.103).

O melhor desempenho do sexo feminino, observado de modo significativo no subteste de escrita do TDE nas duas avaliações, também foi constado nos dados do SAEB de 1999 (Bonamino et al., 2002) e de 2003 (Instituto Nacional de Estudos e Pesquisas Educacionais, 2004): em todas as séries, a média de desempenho em língua portuguesa das alunas foi significativamente maior, seguindo uma tendência internacional vista na avaliação da habilidade de leitura no PISA, desde o ano 2000, segundo Bonamino et al. (2002).

Assim como o subteste de leitura não exigiu nenhuma outra habilidade do aluno a não ser ler 70 palavras, o subteste de escrita exigiu apenas ouvir e registrar as 34 palavras ditadas e o próprio nome; não houve necessidade de contextualizá-las ou promover uma articulação entre elas. Observou-se, no subteste de escrita, um aumento significativo de todos os grupos 
de alunos de um ano para outro; no entanto essa melhora não foi suficiente para atingir a classificação média no TDE. Nesse sentido, o baixo desempenho apresentado pelos alunos nessas duas habilidades revela dificuldades subjacentes ao processo de leitura e escrita.

Da mesma forma que ocorreu na escrita, o desempenho dos alunos no subteste de aritmética mostrou diferenças significativas entre as duas avaliações, mas não a ponto de mudar a classificação inferior. Esse baixo desempenho pode estar respaldado nas diversas dificuldades do ensino da matemática, que vão desde a formação de professores das séries iniciais, as mudanças ocorridas tanto em conceito quanto em metodologia na área da matemática e a própria dificuldade na articulação do conteúdo da matemática e o uso pelo aluno no cotidiano (Novais \& Silva, 2004).

No entanto uma análise intra-série, como se faz no SAEB, mostrou diferença significativa apenas no subteste de aritmética, entre a quarta série de 2001 e a de 2002, indicando que não estaria ocorrendo uma mudança global, de ano para ano, na escola estudada. Esse dado não seria problemático caso o desempenho inicial, em 2001, fosse majoritariamente médio ou superior; mas fica crítico na medida em que o baixo desempenho está sendo mantido. Ocorreram, sim, mudanças no desempenho individual dos alunos ao passar de uma série para outra, mas não foram suficientes para mudar a classificação no TDE.

O baixo desempenho dos alunos encontrado em todas as séries da escola estudada acompanha a tendência no país e no ES, o que permite questionar a qualidade do ensino oferecido a essa população, contrapondo-se à tendência geral de pais e professores responsabilizarem o aluno pelo seu fracasso escolar (Araújo \& Schwartzman, 2002; Neves \& Almeida, 1996). Os dados fornecem ainda indicações para a necessidade de intervenções com essa população, auxiliando professores e psicopedagogos na sua prática profissional.

Convém ainda salientar que os resultados rebaixados no TDE corroboram a noção de que o fracasso escolar é um fenômeno derivado da interação de uma série de fatores (Demo, 1992; Ferreiro, 1992; Leite, 1988; Mello, 1979). Desse modo, o baixo desempenho em escrita, aritmética e leitura pode vir a ser um fator constituinte de um futuro diagnóstico de dificuldade de aprendizagem, caso não sejam fomentados programas de intervenção diferenciados ou mais eficientes do que aqueles propostos pelo sistema de ciclos, a exemplo da recuperação paralela.

Com bases nos dados aqui obtidos, especialmente o intervalo de dois anos para que ocorra alguma diferenciação no desempenho acadêmico, pode-se concordar com Martins (2003) sobre a necessidade de rever como está sendo implantada a progressão continuada de modo que o entrave não continue sendo justamente a colocação em prática de idéias fundamentadas e bem-intencionadas. A metodologia aqui adotada vai de encontro à sugestão de Gomes (2004) a respeito do uso de metodologias diferenciadas na avaliação de investigações da realidade educacional, possibilitando o aprofundamento da análise dos dados de pesquisas nessa área. Por fim, considera-se que os baixos resultados no TDE devem ser analisados como dificuldades geradas, em grande parte, pelas condições de ensino do nosso país, incluindo aqui o processo de avaliação do desempenho da criança.

\section{Referências}

Araújo, J. B. O., \& Schwartzman, S. (2002). A escola vista por dentro. Belo Horizonte: Alfa Educativa.

Barretto, E. S. S., Pinto, R. P., Martins, A. M., \& Duran, M. C. G. (2001). Avaliação na educação básica nos anos 90, segundo os periódicos acadêmicos. Cadernos de Pesquisa da Fundação Carlos Chagas, 114, 49-88.

Bonamino, A., Coscarelli, C., \& Franco, C. (2002). Avaliação e letramento: concepções de aluno letrado subjacentes ao SAEB e ao PISA. Educação e Sociedade, 23 (81), 91-113.

Brasil. (2005). Lei no9.394, de 20 de dezembro de 1996. Estabelece as Diretrizes e Bases da Educação Nacional. Diário Oficial da república Federativa do Brasil, Brasília, 21 de dez. 1996. Acessado em março 20, 2005, disponível em http://www.in.gov.br

Carvalho, M. P. (2001). Estatísticas de desempenho escolar: O lado avesso. Educação e Sociedade, 22 (77), 231-252.

Carvalho, A. E., Linhares, M. B. M., \& Martinez, F. E. (2001). História de desenvolvimento e comportamento de crianças nascidas pré-termo e baixo peso $(<1.500 \mathrm{~g})$. Psicologia: Reflexão e Crítica, 14 (1), 1-33.

Conselho Federal de Psicologia. (Brasil). Edital CFP n ${ }^{\circ} 2$ de $6 /$ 11/2003 - Processo de Avaliação dos Testes Psicológicos. Acessado em dezembro 10, 2004, disponível em http://pol.org.br/arquivos_pdf/edital_efp_n2.pdf 
Demo, P. (1992). Formação de formadores básicos. Em Aberto, 12 (5), 23-42.

Demo, P. (1998). Promoção automática e capitulação da escola. Ensaio: avaliação e políticas públicas em educação. Fundação Cesgranrio, 6(19), 159-190.

Dias, T. B., Enumo, S. R. F., Turini, F. A., \& Ferrão, E. S. (2003), Dificuldade de aprendizagem: avaliação do desempenho em provas acadêmicas e cognitivas. In S. R. F. Enumo, S. S. Queiroz \& A. Garcia (Orgs), Desenvolvimento humano e aprendizagem: algumas análises e pesquisas (pp.59-77). São Paulo: Casa do Psicólogo.

Dias, T. L., Silva, S. R., Enumo, S. R. F., \& Rabbi, J. S. (2005) Análise de erros da escrita em alunos do Ensino Fundamental de Vitória, ES. Vertentes, 25, 29-41.

Ferreiro, E. (1992). Com todas as letras. São Paulo: Cortez.

Ferrão, M., Beltrão, K., \& Santos, D. (2002). Política de não-repetência e a qualidade da educação: evidências obtidas da modelagem dos dados da $4^{a}$ série do SAEB-99. Estudos em Avaliação Educacional, 26, 47-73.

Fonseca, V. (1995). Introdução às dificuldades de aprendizagem. Porto Alegre: Artes Médicas.

Gomes, C. A. (2004). Quinze anos de ciclos no ensino fundamental: um balanço das pesquisas sobre a sua implantação. Revista Brasileira de Educação, 25, 39-52.

Instituto Nacional de Estudos e Pesquisas Educacionais. Resultados do SAEB 2003. Acessado em fevereiro 10,2005, disponível em www.inep.gov.br/download/saeb/2004/ resultados/ Brasil.pdf

Leite, S. A. S. (1988). O fracasso escolar no ensino de $1^{\circ} \mathrm{grau}$ Revista Brasileira de Estudos Pedagógicos, 69 (163), 510-540.

Linhares, M. B. M., Santa Maria, M. R., Escolano, A. C. M., \& Gera, A. A. (1998). Avaliação cognitiva assistida: Uma abordagem promissora na avaliação cognitiva de crianças. Temas em Psicologia, 6 (3), 231-254.

Martins, C. M. C. (2003). Os docentes avaliando o Regime de Progressão Continuada: O primeiro olhar. Um estudo de caso [Resumo completo]. In Associação Nacional de Pesquisa e Pós-Graduação em Educação (Org.), Anais Eletrônicos do XXVI Simpósio Nacional. Poços de Caldas: ANPED. Acessado em fevereiro 28, 2006, disponível em www.anped.org.br/26/posteres/chelseamariamartins.rtf

Marturano, E. M. A. (1999). Recursos no ambiente familiar e dificuldades de aprendizagem na escola. Pesquisa: Teoria e Pesquisa, 15 (2), 135-142.

Medeiros, P. C., Loureiro, S. R., Linhares, M. B. M., \& Marturano, E. M. A. (2000). Auto-eficácia e os aspectos compor- tamentais de crianças com dificuldade de aprendizagem. Psicologia: Reflexão e Crítica, 13 (3), 327-336.

Mello, G. N. (1979). Fatores intra-escolares como mecanismos de seletividade do primeiro grau. Educação e Sociedade, 13 (3), 70-80.

Menezes-Filho, N., Vasconcellos, L., \& Werlang, S. R. C. (2005). Avaliando o impacto da progressão continuada no Brasil [Resumo completo]. In Sociedade Brasileira de Econometria (Org.), Anais do XXVII Encontro Brasileiro de Econometria. Natal: SBE. Acessado em fevereiro 2, 2000, disponível em www.sbe.org.br/ebe27/054.pdf

Neves, M. B. J., \& Almeida, S. F. C. (1996). O fracasso escolar na $5^{a}$ série, na perspectiva de alunos repetentes, seus pais e professores. Psicologia: Teoria e Pesquisa, 12 (2), 147-156.

Neves, E. R. C., \& Burochovitch, E. (2004). A motivação dos alunos no contexto da progressão continuada. Psicologia: Teoria e Pesquisa, 20 (1), 77-85.

Novais, A. B., \& Silva, P. V. (2004). As dificuldades com o ensino de matemática. In Universidade do Estado de Mato Grosso (Org.), I Seminário de Comunicação pública dos trabalhos monográficos de conclusão de curso. Programa Módulos temáticos de formação de professores (p.126). Cáceres: UNEMAT Editora.

Patto, M. H. S. (1990). A produção do fracasso escolar. São Paulo: T. A. Queiroz.

Santa Maria, M. R., \& Linhares, M. B. M. (1999). Avaliação cognitiva assistida de crianças com indicações de dificuldade de aprendizagem escolar e deficiência mental leve. Psicologia: Reflexão e Crítica, 12 (2), 395-417.

Souza, M. P. R. (1997). A queixa escolar e o predomínio de uma visão de mundo. In A. S. Machado \& M. P. R. Souza (Orgs.), Psicologia escolar: em busca de novos rumos (pp.39-63). São Paulo: Casa do Psicólogo.

Stein, L. M. (1994). TDE-Teste de Desempenho Escolar:manual para aplicação e interpretação. São Paulo: Casa do Psicólogo.

Stevanato, I. S., Loureiro, S. R., Linhares, M. B. M., \& Marturano, E. M. A. (2003). Autoconceito de crianças com dificuldades de aprendizagem e problemas de comportamento. Psicologia em Estudo, 8 (1), 67-76.

Wechsler, S. M. (1998). Criatividade: descobrindo e encorajando. Campinas: Ed. Psy.

Recebido em: 11/11/2005

Versão final reapresentada em: 31/3/2006

Aprovado em: 22/6/2006 